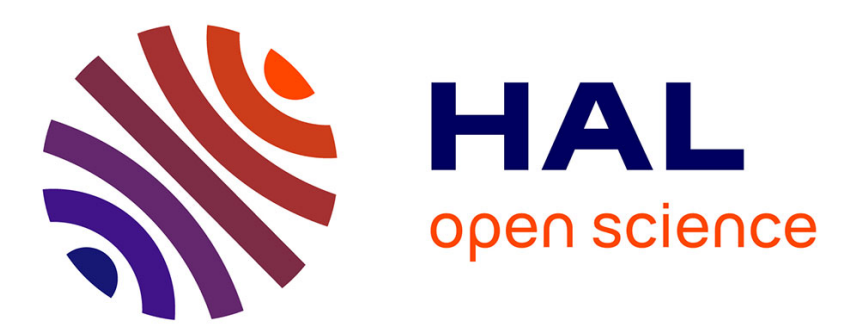

\title{
Pratiques informationnelles des enseignants : le cas des ressources institutionnelles en ligne
}

\author{
Carine Aillerie, Ny aina Rakotomalala harisoa
}

\section{To cite this version:}

Carine Aillerie, Ny aina Rakotomalala harisoa. Pratiques informationnelles des enseignants : le cas des ressources institutionnelles en ligne. Études de communication - Langages, information, médiations, 2020, 54, pp.137-156. 10.4000/edc.10252 . hal-02947893

\section{HAL Id: hal-02947893 \\ https://hal.science/hal-02947893}

Submitted on 24 Sep 2020

HAL is a multi-disciplinary open access archive for the deposit and dissemination of scientific research documents, whether they are published or not. The documents may come from teaching and research institutions in France or abroad, or from public or private research centers.
L'archive ouverte pluridisciplinaire HAL, est destinée au dépôt et à la diffusion de documents scientifiques de niveau recherche, publiés ou non, émanant des établissements d'enseignement et de recherche français ou étrangers, des laboratoires publics ou privés. 


\section{Pratiques informationnelles des enseignants : le cas des ressources institutionnelles en ligne}

Carine Aillerie

Université de Poitiers, EA6316 - Laboratoire TECHNE

Laboratoire de recherche en TECHnologies Numériques pour l'Éducation

carine.aillerie@univ-poitiers.fr

Ny Aina Rakotomalala Harisoa

Direction de la recherche et du développement sur les usages du numérique éducatif

Réseau Canopé - Chasseneuil du Poitou

nyaina.rakotomalala@reseau-canope.fr

Si le métier d'enseignant peut en premier lieu se définir par la présence physique dans une classe et par les interactions avec des élèves, une grande partie se déroule cependant en dehors de la classe, le plus souvent selon des modalités individuelles, mobilisant équipement et documentation personnels. Ce travail à la maison s'effectue y compris sur le temps libre, voire pendant les vacances (Gueudet et Trouche, 2008 ; ministère de l'Éducation nationale, 2016). Cette face « cachée » du métier se concentre sur la lecture et l'appropriation des textes officiels, l'actualisation et la diversification des connaissances disciplinaires, ainsi que sur la préparation des cours. Elle implique le recours à une documentation multiple et suppose des opérations de recherche, de sélection, d'exploitation, de stockage des informations collectées, aboutissant à la production de documents professionnels (fiches élèves, notes et formalisations de séances pédagogiques, documents d'évaluation, etc.). Ces pratiques informationnelles enseignantes et leurs évolutions demeurent encore en partie méconnues (Diekema et Olsen, 2014). Nous assistons en effet, depuis une dizaine d'années, à une modification notable du paysage informationnel à disposition de la population en général et donc des enseignants, tant du point des pratiques personnelles que des pratiques professionnelles. On peut ainsi noter la multiplication des portails et bases de données en ligne, des plateformes collaboratives et sociales, la complexification des modes de stockage et de partage de l'information. On peut également noter, pour ce qui concerne plus spécifiquement l'éducation, l'offre en déploiement de ressources pédagogiques privées ou libres, la généralisation des plateformes de partage de contenus, des sites web de communautés ou blogs personnels d'enseignants. Qui plus est, la maîtrise de cet environnement hétéroclite semble conditionner très directement l'exercice du métier, tel qu'il est défini par son ministère de tutelle, lorsqu'il «s'agit de faire évoluer ses cours grâce au numérique et en actualisant ses propres connaissances ${ }^{1} \gg$.

$\mathrm{Au}$ vu de la fonction centrale occupée par le maniement de ressources dans le métier d'enseignant, il paraît donc toujours nécessaire d'interroger la réalité de ces pratiques informationnelles. C'est à ce chantier que se propose de contribuer cet article, s'appuyant sur une étude mettant en œuvre une méthodologie croisée auprès d'enseignants des premier et second degrés, usagers d'une offre en ligne de ressources institutionnelles d'accompagnement à la mise en œuvre des programmes scolaires (nouveaux programmes de l'école et du collège de 2015). Cette étude répond à un objectif opérationnel, au service de l'institution pourvoyeuse de ces ressources, qui vise à cerner les représentations des enseignants à l'égard de ces

\footnotetext{
${ }^{1}$ http://www.devenirenseignant.gouv.fr/cid98754/etre-enseignant-aujourd-hui.html
} 
ressources et à en comprendre les usages effectifs. Les résultats obtenus permettent également de préciser la place de ces ressources institutionnelles et leur spécificité en tant que telles dans les pratiques informationnelles professionnelles et les représentations des enseignants interrogés. Dans cet article, nous proposons dans un premier temps de définir les notions théoriques sur lesquelles se base notre travail, notamment l'articulation entre pratiques informationnelles et culture de l'information. Nous expliciterons ensuite nos choix méthodologiques incluant à la fois une démarche quantitative et qualitative. Y seront présentés les modalités de recueil de données et la complémentarité des démarches choisies. La dernière partie éclairera les modalités de consultation des ressources institutionnelles par les enseignants, sur la place qu'ils accordent à ces ressources, sur les spécificités de celles-ci et sur les différentes dimensions entrant en jeu dans la construction de leurs pratiques informationnelles.

\section{Articulation entre ressources, pratiques informationnelles et représentations}

Dans un premier temps, il convient de définir les termes « ressources », «pratiques informationnelles» et «représentations» mobilisés dans cet article. Ces éléments nous permettront de préciser la question de recherche énoncée en introduction.

\subsection{Qu'est-ce qu'une ressource pour l'enseignement?}

Le terme « ressources » est polysémique, souvent disqualifié de par son imprécision (Baron et Dané, 2007). D’une manière générale, les ressources englobent tous les éléments susceptibles d'enrichir concrètement le travail de l'enseignant, voire d'influencer ses pratiques et sa réflexion professionnelles (Adler, 2010 ; Besnier, 2016). Reverdy identifie trois catégories aptes à préciser la nature de ces ressources : les ressources «matérielles », les ressources « humaines » et les ressources « culturelles» (Reverdy, 2014). Les ressources «matérielles » incluent les ressources imprimées (manuels scolaires, articles de revues professionnelles, textes authentiques étudiés en langues, etc.), mais aussi les ressources numériques (sites institutionnels ou disciplinaires, plateformes en ligne, etc.) ainsi que les objets de la vie quotidienne pouvant être utilisés à des fins pédagogiques (des photos de vacances par exemple). Les ressources « humaines » sont constituées par les savoirs professionnels des enseignants, acquis au gré de l'expérience ou des formations, les échanges entre pairs ou autres acteurs éducatifs. Les ressources «culturelles» se réfèrent aux éléments de la forme scolaire proprement dite (emplois du temps, didactisation des disciplines, vocabulaire, etc.).

Dans le prolongement de ces réflexions, nous employons ici le terme « ressources » dans le sens que lui confère Loffreda en tant qu' " entité matérielle ou numérique qui est actualisée par la pratique de l'enseignant» à finalité spécifique d'enseignement et mobilisant «trois éléments centraux liés aux personnes : les valeurs, les intérêts personnels, et tout ce qui relève de la construction professionnelle » (Loffreda, 2017, 6). L'objet de notre étude se rapporte à des ressources institutionnelles mises en ligne à l'occasion de la rénovation des programmes scolaires. Celles-ci se composent essentiellement de textes, mais comportent également des images fixes et animées. Utilisées par les enseignants dans le but d'opérationnaliser dans leurs classes le programme scolaire prescrit, ces ressources institutionnelles incluent le programme lui-même ainsi que les documents qui l'accompagnent, en précisent la teneur et les orientations, fournissent des exemples de mises en œuvre concrètes (Ball et Cohen, 1996). Ces ressources viennent étayer l'une des missions de l'enseignant qui consiste en l'interprétation de ce curriculum prescrit (Perrenoud, 1993) en vue de son adaptation à une situation d'enseignement donnée. Cette opération est d'autant plus prégnante dans le contexte de réforme dans lequel s'inscrit ce travail. 
Il faut retenir ici que la «ressource» au service de la pratique enseignante est multiforme. Qualifiée comme telle en fonction de ses besoins par l'enseignant, elle mêle contenus liés à la vie de tous les jours et matériel spécifiquement dédié à la pratique professionnelle. C'est dans ce paysage informationnel hétéroclite que viennent s'inscrire les pratiques liées aux ressources institutionnelles concernées par notre étude.

\subsection{Pratiques informationnelles et culture de l'information}

Nous prenons en compte les apports de l'approche documentaire du didactique, attachée à l'étude du travail documentaire des enseignants en contexte numérique (Gueudet et Trouche, 2008). Nous situons cependant directement notre propos dans la lignée des réflexions qui, en sciences de l'information et de la communication, travaillent l'ancrage conceptuel de la «pratique» informationnelle en articulation avec la compréhension des usages de l'information, entendus comme les diverses manifestations empiriques de cette pratique (Gardiès, Fabre et Couzinet, 2010). À l'initiative d'un numéro spécial d'Études de communication, Chaudiron et Ihadjadène formalisent la pratique informationnelle comme :

la manière dont l'ensemble de dispositifs, de sources, d'outils, de compétences cognitives sont effectivement mobilisés dans les différentes situations de production, de recherche, traitement de l'information. Nous englobons dans ce terme de «pratiques» les comportements, les représentations et les attitudes informationnelles de l'humain (individuel ou collectif) associés à ces situations (Chaudiron et Ihadjadène, 2010).

Cette définition est largement reprise dans les écrits consacrés à la pratique informationnelle en milieux scolaire et universitaire, qui entérine le rôle central de l'acteur. Elle se rapporte ainsi à la culture de l'information telle que conceptualisée notamment par Juanals (2003), ne se résumant pas à un ensemble de compétences ou de comportements mais détenant une dimension éthique et sociale. Dans le prolongement de décennies de recherches consacrées à l'activité informationnelle et aux usages de l'information, ce sont «l'épaisseur » des pratiques et les conditions au principe de leur «construction» (Souchier, Le Marec et Jeanneret, 2003) qui mobilisent aujourd'hui la recherche et qui nous intéressent ici. Cette construction des pratiques, dans le cas des enseignants qui nous occupent, est à replacer dans le champ de l'exercice de la profession et des représentations individuelles et collectives qui sont liées à cette profession et aux cadres institutionnels qui la régissent.

\subsection{Pratiques et représentations}

Les pratiques informationnelles recoupent des comportements observables ainsi que des représentations mentales et sociales. En psychologie, la représentation est « un ensemble organisé d'opinions, d'attitudes, de croyances et d'informations se référant à un objet ou une situation. Elle est déterminée à la fois par le sujet lui-même (son histoire, son vécu), par le système social et idéologique dans lequel il est inséré, et par la nature des liens que le sujet entretient avec ce système social » (Abric, 1993). Dans cet article, nous interrogeons les valeurs et les significations attribuées par les enseignants à ces ressources institutionnelles dans le cadre de l'inscription de ces ressources dans leurs écosystèmes informationnels personnels et collectifs. Les pratiques informationnelles de l'enseignant sont directement liées à ses représentations et valeurs professionnelles. En retour, ses expériences quotidiennes avec l'information sont susceptibles de faire évoluer ses représentations, voire ses pratiques, professionnelles. Ainsi cette imbrication entre représentations et pratiques est-elle fondamentale dans la constitution d'une culture de l'information de l'enseignant.

\section{Une démarche méthodologique mixte pour recenser et comprendre les pratiques}




\subsection{Recenser des pratiques : une enquête par questionnaire (janvier — février 2017)}

Afin d'apporter des éléments de réponse à notre questionnement, nous avons mis en œuvre un protocole méthodologique croisé, impliquant une enquête par questionnaire et des entretiens qualitatifs auprès d'enseignants français volontaires. Le travail quantitatif a précédé le travail qualitatif. Si l'enquête par questionnaire avait pour objectif d'identifier et de recenser les pratiques des enseignants vis-à-vis de ces ressources, les entretiens qualitatifs nous ont permis d'approfondir le sens de ces pratiques pour nos répondants.

Comportant 26 questions, l'enquête par questionnaire est structurée autour de trois parties : profil des répondants, pratiques, perceptions des enseignants. Le lien vers l'enquête a été déposé sur la page d'accueil du portail hébergeant les ressources. Il a également été diffusé par messagerie électronique aux enseignants des niveaux scolaires visés (du niveau primaire au niveau lycée). 9611 enseignants ont répondu au questionnaire, qui déclarent avoir consulté au moins une fois l'offre de ressources. C'est sur cette base qu'a porté notre analyse statistique (tableau 1).

\begin{tabular}{lcc}
\hline & Effectif & Pourcentage \\
Profil & 5659 & $59 \%$ \\
Enseignant en collège & 3684 & $38 \%$ \\
Professeur des écoles (y compris enseignant spécialisé) & 185 & $2 \%$ \\
Enseignant en lycée général et technologique & 83 & $1 \%$ \\
Enseignant en lycée professionnel & & \\
\hline Type d'établissement & 8151 & $85 \%$ \\
Public & 1424 & $15 \%$ \\
Privé & 36 & $0 \%$ \\
Ne sait pas & & \\
\hline Situation géographique de l'établissement & 5696 & $59 \%$ \\
En milieu urbain & 3768 & $39 \%$ \\
En milieu rural & 147 & $2 \%$ \\
Ne sait pas & & \\
\hline Ancienneté dans l'éducation & 1456 & $15 \%$ \\
3 ans ou moins & 2078 & $22 \%$ \\
De 4 à 10 ans & 3379 & $35 \%$ \\
De 11 à 20 ans & 1835 & $19 \%$ \\
De 21 à 30 ans & 863 & $9 \%$ \\
Plus de 30 ans & & \\
\hline
\end{tabular}

Tableau 1 : Caractéristiques des répondants $(n=9611)$

\subsection{Comprendre ces pratiques : des entretiens qualitatifs (mars -avril 2017)}

Quatre entretiens collectifs en présentiel sont venus compléter et enrichir les résultats obtenus par questionnaire. Ces entretiens ont eu lieu à l'issue de la phase d'analyse des données quantitatives et organisés en fonction des résultats de cette analyse. Au vu de la littérature, il nous a ainsi semblé utile d'interroger les enseignants en fonction de leur niveau d'enseignement et en fonction de leur discipline. N'ayant pas la possibilité matérielle de constituer de multiples groupes disciplinaires étoffés, nous avons fait le choix de réunir les enseignants du secondaire en fonction de l'orientation générale de leur discipline. Ainsi, pour chaque entretien, les participants étaient répartis comme suit : 6 enseignants du premier degré (cycles 2 et 3), 7 enseignants du second degré (sciences et technologie), 9 enseignants du second degré (sciences 
humaines, lettres, langues et arts). Les entretiens ont été menés sur la base d'un conducteur. Les propos ont été intégralement retranscrits, catégorisés et analysés au regard de notre question de recherche.

\section{Résultats}

Nos résultats agrègent les analyses issues des deux volets méthodologiques complémentaires. Il faut d'emblée souligner une concordance des réponses issues de l'enquête avec les propos tenus en entretiens ainsi qu'une homogénéité des résultats entre enseignants du premier et du second degré, toutes disciplines confondues.

\subsection{Des modalités de consultation largement individuelles}

La fréquence de consultation des ressources étudiées est assez faible : $55 \%$ des 9611 répondants les compulsent au plus une fois par mois. En revanche, la durée de consultation de ces ressources est plutôt longue. $40 \%$ des 9611 enseignants passent plus de 20 minutes à la lecture de ces ressources et $22 \%$ entre 15 et 20 minutes. Les ressources d'accompagnement des nouveaux programmes relatives aux cycles 3 et 4 ont été les plus consultées.

$77 \%$ des 9611 répondants lisent les ressources sur l'écran de leur ordinateur et $73 \%$ les impriment. Les téléphones portables, les tablettes et les liseuses sont très peu utilisés pour consulter ces ressources. Ces dispositifs semblent peu compatibles avec la longueur et la densité des ressources, et surtout peu conciliables avec les pratiques exprimées de conservation, de réutilisation, de modification.

L'impression est notamment privilégiée dans le cas de partage de ressources ou d'utilisation à court terme. L'inconfort de la lecture à l'écran et les modalités d'appropriation du document (annotation, surlignage, etc.) constituent des arguments souvent avancés pour justifier l'impression : «j'ai besoin de papier, posé » (enseignant du $1^{\text {er }}$ degré), « je lis sur l'écran. Mais au-delà de 2-3 pages, j'ai besoin d'avoir la possibilité de revenir en arrière et de prendre des notes, et là, j'ai besoin d'imprimer », « on peut aller le chercher n'importe quand, sans avoir à ouvrir son ordi » (enseignants du second degré groupe sciences et techniques).

L'enregistrement des ressources se fait au fur et à mesure de la consultation. Les ressources enregistrées font l'objet de réorganisations personnelles : les fichiers sont renommés et les arborescences repensées. Les enseignants interrogés reconnaissent parfois se perdre dans leur propre organisation, mais ils recourent plus facilement à ces archives qu'au site (risque de ne pas retrouver la ressource ou de rencontrer des problèmes de connexion) : «toutes les ressources, je les télécharge, en fait, et je les mets dans mon propre archivage qui est plus cohérent et plus simple», «du coup, nous, on se crée son propre système d'archivage...» (enseignants du second degré groupe humanités).

Les enseignants consultent majoritairement ces ressources chez eux, au calme, et à partir de leur ordinateur personnel. Les modalités de consultation de ces ressources sont toujours relatives au temps dont ils disposent pour ce faire. Il s'agit notamment du temps de repérage des ressources possiblement utiles, de la lecture attentive et de la compréhension ainsi que de la collecte et de l'enregistrement des ressources : «C'est quelque chose que je vais consulter quand j'ai du temps, pas quand j'en ai besoin » (enseignant $1^{\text {er }}$ degré), «j'ai tellement à digérer tout seul d'abord [...] chez moi, tranquille, quand j'ai le temps » (enseignant du second degré groupe sciences et techniques), «Et ça cogite en même temps, [...] ça demande du calme et du temps » (enseignant du second degré groupe sciences et techniques).

La dimension temporelle apparaît cruciale dans la construction des pratiques informationnelles des répondants, l'emploi du temps prenant ici quasiment le pas sur le besoin d'information en tant que tel, qui se trouve en quelque sorte différé. Ce temps est lié non seulement à l'assimilation des programmes, mais plus fondamentalement, à un processus 
réflexif sur la pratique professionnelle : «c'est là que la digestion est difficile, en fait », « la remise en cause de sa pratique que ça induit » (enseignant premier degré). Cette démarche est donc plutôt présentée comme individuelle, voire solitaire : «j'avais besoin d'être seul pour essayer de comprendre » (enseignant du second degré groupe sciences et techniques).

\subsection{Des ressources institutionnelles pour nourrir la culture professionnelle}

Les ressources étudiées sont mobilisées par nos répondants afin de prendre connaissance des programmes et dans le cadre de la préparation des cours. C'est en troisième lieu une perspective d'autoformation qui se dégage des réponses, en particulier pour le premier degré.

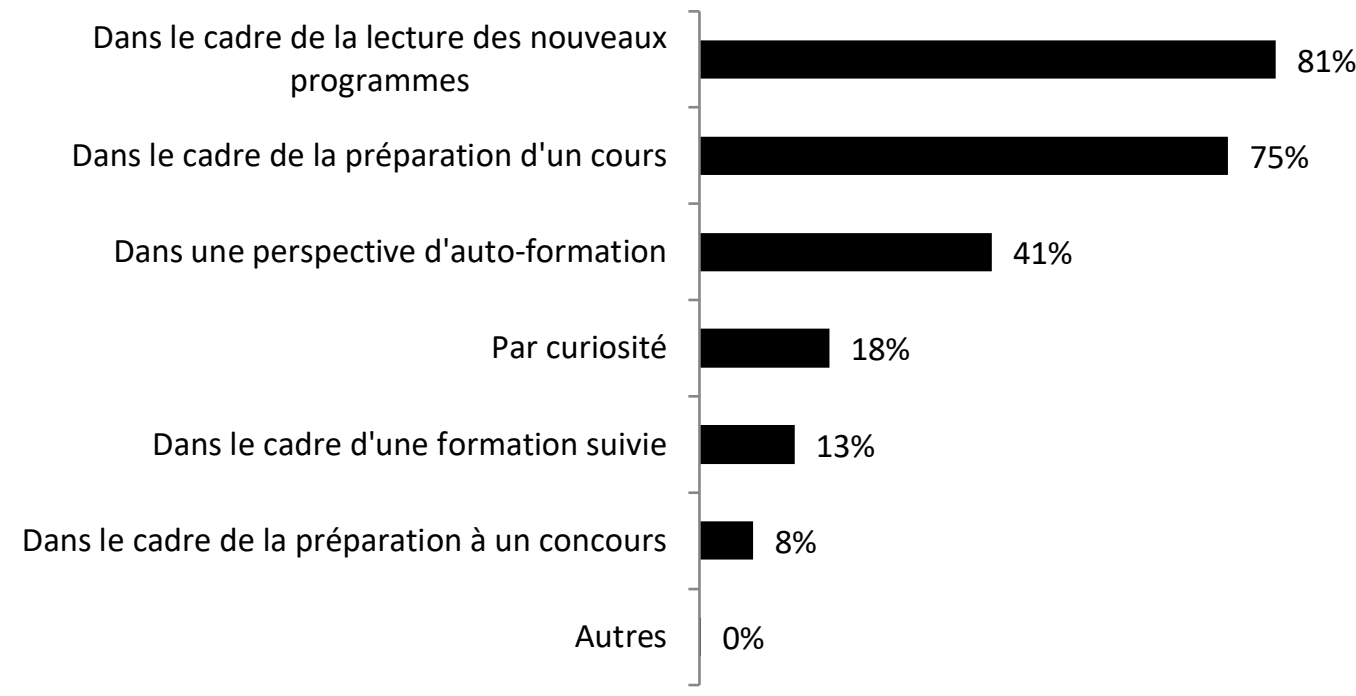

Graphique 1 : Motifs de consultation des ressources d'accompagnement sur le site institutionnel $(n=9611)$

La motivation des répondants à consulter les ressources étudiées est surtout celle de la culture professionnelle: mieux connaître la réforme, se tenir au courant des avancées pédagogiques, avoir des éclairages sur l'approche prônée par les nouvelles exigences : «Pour être à la page » (enseignant du $1^{\text {er }}$ degré), « c'est vraiment pour voir ce qu'ils veulent vraiment » (enseignants du $2^{\text {nd }}$ degré groupe Humanités). Pour certains, c'est la réforme elle-même qui a suscité leur curiosité à l'égard de ces ressources.

Les motivations pour consulter ces ressources sont ainsi également opératoires. Selon ces enseignants, comprendre l'esprit de la réforme, c'est aussi avoir une idée précise de sa mise en œuvre dans la classe : «Qu'est-ce que je dois faire ? Comment je vais faire ? » (enseignant du $1^{\text {er }}$ degré), «Ce n'est pas forcément pour prendre un exemple tel quel, mais vraiment à la recherche des idées » (enseignant du $2^{\text {nd }}$ degré groupe Humanités). Ils sont nombreux à y chercher des repères quant à ce qu'ils doivent et peuvent réellement exiger de leurs élèves. Ils sont ainsi à la recherche de progressions, d'outils pédagogiques concrets, d'exemples réalistes, c'est-à-dire applicables dans leurs contextes d'enseignement, ainsi que de ressources à utiliser en classe (photos, vidéos, affiches, etc.). Cette dimension conditionne la sélection et la lecture des ressources. Il ne s'agit pas de trouver des séances "clé en main » mais de disposer de ressources adaptables à leurs objectifs pédagogiques. Ce processus se fait, là encore, sur un temps long et est en lien direct avec la réflexivité professionnelle de l'enseignant.

\subsection{Une forte spécificité institutionnelle des ressources étudiées}


$68 \%$ des 9611 répondants ont pris connaissance de l'existence de ces ressources en se rendant directement sur le site hébergeur. C'est la nature proprement institutionnelle de cette offre de ressource qui les intéresse et qui motive la consultation. Dans le détail, au sein de cette catégorie, $79 \%$ des 3684 professeurs des écoles, $74 \%$ des 5659 enseignants en collège, $80 \%$ des 185 enseignants en lycée général et technologique et $78 \%$ des 83 enseignants en lycée professionnel ayant répondu à notre enquête accordent leur confiance à ces ressources précisément du fait qu'elles soient hébergées par un site institutionnel : «j'y allais vraiment pour de l'institutionnel» (enseignants du second degré groupe Humanités), «ce qui est ministériel, d'abord. Ce qui est officiel, en tout premier » (enseignant du premier degré). À ce titre, le portail hébergeur est directement ciblé dans les moteurs de recherche commerciaux.

Les enseignants se tournent vers les ressources institutionnelles en premier lieu de par le haut degré de fiabilité qu'ils leur accordent, en termes de validité de l'information comme en termes de conditions juridiques d'utilisation de la ressource. Il s'agit également d'obtenir une forme de traduction des programmes ou de commentaire officiel sur ces programmes et sur leur mise en œuvre. À ce titre également, ils soulignent leur attachement à l'identification des auteurs de ces ressources, notamment de manière à les rattacher au discours soit d'un pair soit d'un prescripteur.

\subsection{Le rôle crucial des temps de partage}

Du point de vue de la connaissance des ressources, on note l'importance des temps de formation (initiale ou continue), des relais de communication institutionnels (sites académiques notamment) et de la communication par mail. Les réseaux sociaux n'occupent ici qu'une faible place : seulement $2 \%$ des 9611 répondants déclarent avoir connu l'existence de l'offre de ressources par ce biais. En effet, dans un premier temps, la seule mise à disposition de la ressource ne suffit pas pour provoquer l'usage. Dans un deuxième temps, et dans la perspective de la réflexion professionnelle, l'usage ne se limite pas à la consultation des ressources mais implique la modification de la ressource par l'adaptation à un contexte d'enseignement donné et à une pratique professionnelle existante. Cela passe par le partage et la discussion entre pairs, pendant de l'appropriation solitaire.

C'est également le rôle de l'institution (inspecteurs, conseillers pédagogiques et formateurs) qui se confirme ici comme étant fondamental. Beaucoup de répondants déclarent en effet avoir eu connaissance de cette offre de ressources à l'occasion d'une information ou d'une formation institutionnelles : animation pédagogique, présentation des ressources, courriers électroniques ciblés de la part des corps d'inspection. Ce sont le plus souvent les ressources les plus directement utilisables pour la classe qui semblent avoir été alors mises en lumière. Ces relais institutionnels sont appréciés et plébiscités, comme cela est apparu dans les réponses au questionnaire et lors des entretiens. Les enseignants expriment leur souhait de pouvoir être accompagnés dans l'usage de ces ressources, de façon à non seulement les connaître mais à les utiliser en classe et à les intégrer dans leur pratique. Cela passe par des temps de travail entre pairs : « ̀̀ la place d'une autoformation, avec un temps personnel difficile, voire impossible à trouver, je souhaiterais plutôt des formations en groupe avec des personnes-ressources ou bien des temps réguliers d'échange et de découverte prévus à l'école avec des collègues $[\ldots] \gg$ (professeur des écoles).

\subsection{Les autres ressources consultées par ces enseignants}

La question des pratiques et représentations des enseignants à l'égard d'une offre de ressources institutionnelles en ligne se pose dans le contexte plus général du paysage informationnel à leur disposition. Comme rappelé en introduction, l'offre est pléthorique. Les 
répondants se disent perdus face à la masse d'information, y compris à l'intérieur du seul périmètre institutionnel. Le temps nécessaire à la recherche et à la lecture s'ajoute à la charge de travail : « [...] Je suis perdu sous le déluge d'information. » (enseignant en collège), « J'y passe beaucoup de temps et me dis toujours que je passe à côté de quelque chose n'ayant pas pu lire tout l'ensemble» (professeur des écoles). Aussi avons-nous interrogé nos répondants quant aux ressources et sources d'informations mobilisées dans le cadre de leur pratique professionnelle, en dehors de l'offre institutionnelle objet principal de notre étude. Comme le montre le graphique (2) ci-dessous, deux éléments se détachent : les sites académiques et les sites ou blogs personnels d'enseignants :

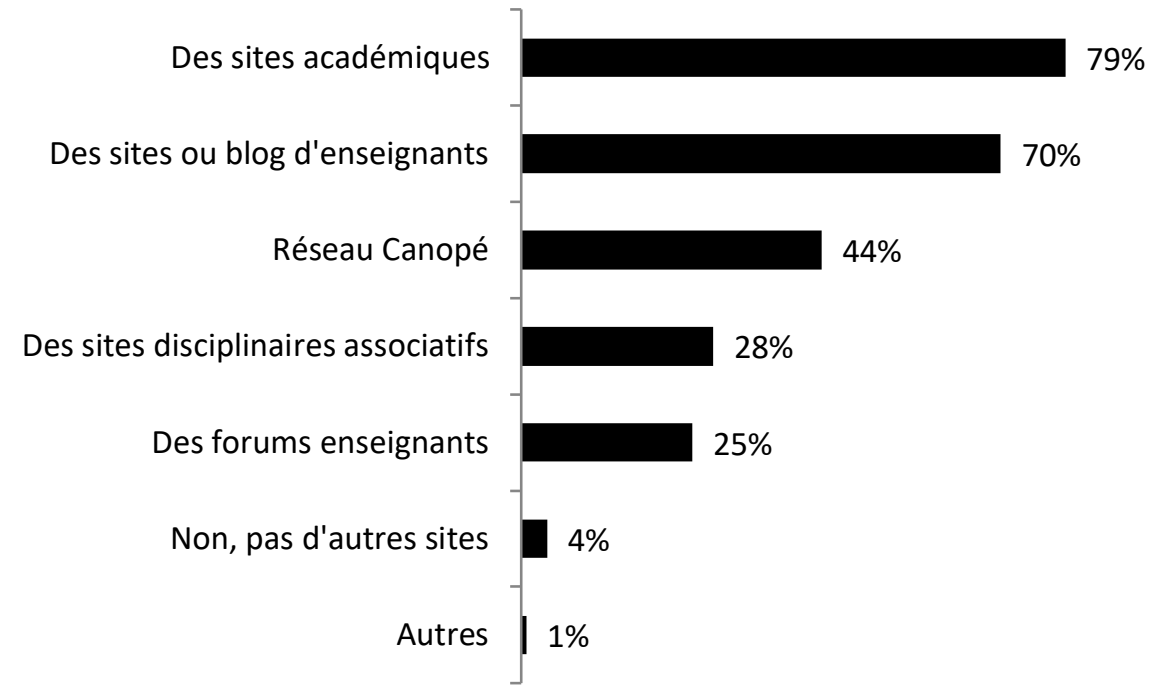

Graphique 2 : Autres sources d'informations visitées par les répondants relatives aux nouveaux programmes $(n=9611)$

\section{Discussion}

La question posée par notre étude peut être ainsi résumée : «quelles inscriptions d'une offre de ressources institutionnelles en ligne dans les pratiques informationnelles professionnelles des enseignants ? » Les résultats précédemment exposés concernent donc des ressources bien spécifiques, qui peuvent être replacés dans la perspective des travaux centrés sur les pratiques informationnelles des enseignants et, notamment, sur les éléments qui orientent le choix des ressources. À la croisée de problématiques communicationnelles et didactiques, ces travaux sont essentiellement menés en sciences de l'information et de la communication et en sciences de l'éducation. Notre étude renvoie aux processus de recherche, d'identification des sources et de traitement de l'information, mais elle est indissociable des processus d'identification professionnelle de l'enseignant et de la communauté dans laquelle il s'inscrit. C'est en ce sens qu'au-delà de la description des usages, notre étude contribue à dessiner les contours d'une culture de l'information enseignante, articulant un ensemble de connaissances relatives à la maîtrise de l'information et un rapport à l'information au cœur des identités professionnelles enseignantes, a fortiori dans un contexte professionnel marqué par les injonctions institutionnelles et les interactions entre pairs. Si les pratiques informationnelles des enseignants constituent un objet de recherche à part entière, la question de la place des ressources institutionnelles dans ces pratiques en constitue une problématique bien spécifique du fait de leur caractère descendant. Il n'est pas suffisant de supposer un usage systématique car obligatoire : encore faut-il interroger la réalité de ces pratiques, ce que nous avons proposé de faire dans cet article. 


\subsection{Dimension individuelle et temporelle des pratiques informationnelles des enseignants}

Nos résultats signalent une forte présence du numérique dans les pratiques informationnelles de nos répondants, le rôle important des moteurs de recherche, la faible fréquentation des banques de ressources en ligne et des comportements de recherche plutôt aléatoires et opportunistes. Le support papier reste cependant très important, dans cette accumulation des ressources collectées. Ce type de constat est également formulé dans le cadre du projet de recherche ANR-ReVEA, notamment dans le cadre de la constitution de classeurs et des documents distribués aux élèves (Messaoui, 2016).

Nos résultats mettent en lumière des habitudes de consultation, de sélection et de lecture largement individuelles, dans le sens où ces habitudes ne sont que très peu collectives, au sens de la collaboration ou coopération avec les pairs. D'autres auteurs signalent cette forte dimension personnelle des ressources mobilisées et des collections que les enseignants se constituent et organisent au fur et à mesure de leurs besoins (Diekema et Olsen, 2014).

L'importance des pratiques de conservation papier et d'impression est à rapprocher de cette dimension individuelle et à long terme des dynamiques d'appropriation de l'information sélectionnée. Les enseignants interrogés décrivent des pratiques de conservation, de réutilisation, de modification de ressources, de réorganisations personnelles des architectures, indissociables du support physique et du temps long lié au temps personnel et à l'espace privé. Les pratiques informationnelles décrites par notre étude, au service d'une pratique en classe, comportent une forte dimension opérationnelle. Ce sont les programmes scolaires et leurs évolutions ainsi que le(s) niveau(x) enseigné(s) qui conditionnent sans surprise la mobilisation des ressources. Les résultats de recherche sur le sujet nous renseignent plus largement sur le fait que les ressources sont mobilisées et les dispositifs intermédiaires qui en permettent l'accès, consultés, dans la mesure où ils sont accessibles, utilisables et susceptibles de contribuer directement à la construction d'une activité ou d'une progression pédagogique (Margolinas et Wozniak, 2010 ; Endrizzi, 2012). Mais cette dimension opérationnelle de leurs pratiques informationnelles renvoie précisément à la réflexivité professionnelle des enseignants interrogés qui ne sont pas en attente de documents "clé en main », mais de ressources modifiables et adaptables à leurs objectifs et à leur contexte d'enseignement, en fonction des exigences attendues par l'institution.

\subsection{Spécificité sociale des ressources institutionnelles}

Les pratiques informationnelles décrites par notre étude apparaissent peu marquées par le travail collectif. L'individualité des pratiques intègre par ailleurs une forte dimension sociale, liée à l'expérience individuelle de l'enseignant ainsi qu'aux autres acteurs et à l'institution (Gardiès et al., 2010). Les travaux de Diekema et Olsen $(2012,2014)$ insistent ainsi sur la dimension personnelle des collections mais aussi sur l'importance de la recommandation par les pairs de ressources ayant été validées et étoffées par une expérience pédagogique antérieure ainsi que sur le rôle des premières années d'enseignement.

Dans le contexte de notre étude, le choix d'une source institutionnelle apparaît comme très important, basé sur la fiabilité perçue des ressources et, précisément, la possibilité d'avoir été testées ou approuvées par des prescripteurs ou des pairs. Il s'agit d'estimer, voire d'opérer, le degré d'adéquation entre les injonctions institutionnelles et les pratiques professionnelles individuelles. C'est la nature institutionnelle de la ressource concernée par notre étude qui intéresse nos participants et qui motive la consultation. Cette consultation implique la modification de la ressource par l'adaptation à un contexte d'enseignement et à une pratique 
professionnelle existante. Il est ainsi question d'autoformation et non pas seulement de maîtrise des programmes et de préparation de cours.

Pour ce qui concerne donc très spécifiquement les ressources institutionnelles, a fortiori lors d'un changement de programme, il faut noter la forte contrainte à l'usage qui ne présage pour autant pas du degré d'acceptation de la ressource. D'après Remillard, Herbel-Eisenmann et Lloyd (2009, cités par Reverdy, 2014), l'usage des ressources institutionnelles par les enseignants dépend étroitement du profil de ces enseignants en termes de représentations de la discipline, du métier et de la marge d'interprétation du curriculum qu'ils s'accordent. Selon la synthèse de Reverdy, l'utilisation effective des ressources proposées via les portails institutionnels, doivent ainsi atteindre le délicat équilibre entre visibilité et invisibilité : « c'està-dire à la fois visibles (ou disponibles) pour être utilisées et améliorer la pratique professionnelle et invisibles pour pouvoir s'effacer au profit de l'apprentissage des élèves » (Reverdy, 2014).

\subsection{Pratiques informationnelles enseignantes et culture de l'information}

Le développement des pratiques numériques enseignantes vient modifier et renforcer des habitudes de recherche, de stockage et de personnalisation des ressources. Nos résultats vont ainsi dans le sens de la définition de l'activité informationnelle enseignante comme un processus itératif qui consiste à « rassembler des ressources, les sélectionner, les transformer, les recomposer, les partager, les mettre en œuvre, les réviser... [...] pour un objectif didactique donné » (Gueudet et Trouche, 2010). Cette activité est cependant devenue exigeante, en termes de temps, d'équipement comme en termes de compétences, informationnelles et numériques lorsqu'il s'agit d'explorer des outils de recherche, de localiser, enregistrer voire modifier des ressources en ligne (Dumouchel et Karsenti, 2017). Nos répondants se disent effectivement perdus face à la masse d'information et de ressources potentielles.

La littérature présente des pratiques informationnelles enseignantes dépendant de variables contextuelles, liées aux ressources à disposition (accessibilité, qualité des ressources) et liées aux conditions d'exercice (profil de l'établissement, équipement à disposition, rôle des personnels de direction par exemple) ainsi qu'à l'emploi du temps (Remillard, 2013). Diekema et Olsen (2011) et Loffreda (2017) soulignent ainsi l'importance du rôle joué par le contexte d'enseignement dans les pratiques informationnelles des enseignants lors de la sélection, de l'organisation, de la conservation et de l'appropriation des informations. Selon ces auteurs, le niveau enseigné, la taille de la classe, le programme, la discipline, les matériels et les ressources existants constituent des paramètres qui conditionnent ces pratiques.

Notre étude a permis de souligner la forte dimension individuelle, voire solitaire, des pratiques informationnelles de nos participants. Gueudet et Trouche insistent pour leur part sur la dimension centrale de la pratique informationnelle «dans l'activité professionnelle des professeurs. [qui] [...] habite tous les aspects de cette activité, tous ses lieux, tous ses temps » (Gueudet et Trouche, 2010). Du point de vue des profils individuels des enseignants comme du point de vue de leurs contextes d'enseignement, leurs pratiques informationnelles s'exposent, en tant que telles, à une diversité voire une hétérogénéité interindividuelle prégnante. Ces pratiques, et donc leur diversité, sont de plus peu visibles, relevant des préparations en dehors de la classe, souvent dans la sphère privée. Elles renvoient à ces « compétences incertaines ou peu reconnues » (Barrère, 2002) par l'institution, même si indispensables à l'exercice professionnel.

C'est cette invisibilité d'une activité centrale dans l'exercice du travail qui a également été pointée en contexte organisationnel, déclinée sous la forme de tâches multiples (recherche, lecture, rédaction, annotation, validation, partage, etc.), nécessitant chacune des compétences particulières à la fois techniques et sociales (Guyot, 2009), engageant ici le rapport de l'acteur 
aux savoirs à enseigner et à l'institution qui le charge de les transmettre. C'est en ce sens que nos résultats se rapportent à une culture de l'information, les pratiques informationnelles décrites étant vitales à l'activité professionnelle enseignante et engageant la construction de l'identité professionnelle des acteurs au sens de Dubar et Tripier (2005). Ces processus de structuration articulent des dynamiques évolutives et paradoxales, à la fois très personnelles et éminemment sociales, relevant d'éléments constants et d'incertitudes. La nature institutionnelle de l'offre de ressource concernée par notre étude permet précisément de travailler cette marge de manœuvre des acteurs, entre logiques informationnelles individuelles et normativité.

\section{Conclusion}

Nos résultats entrent en résonance avec les travaux de recherche attachés à décrire de manière générale les pratiques informationnelles des enseignants. Ils se rapportent à une étude au périmètre limité à une offre bien spécifique de ressources mais font cependant écho à un champ de recherche qui permet d'en évaluer la portée. Nos résultats vont ainsi dans le sens de ceux formulés par d'autres chercheurs et viennent spécifier les usages des ressources institutionnelles par les enseignants, ce type de ressources occupant une place tout à fait particulière dans le paysage informationnel à leur disposition. Nos résultats soulignent également la dimension implicite de la pratique informationnelle de l'enseignant.

Centrale pour le métier, la pratique informationnelle de l'enseignant engage son autonomie et s'impose comme un des lieux d'exercice de sa liberté pédagogique. Un travail sur la ressource institutionnelle et sa spécificité en tant que telle contribue ainsi à interroger la place de ces pratiques informationnelles dans la culture et l'identité professionnelles enseignantes.

Nous pointons l'invisibilité des situations individuelles dans la gestion de tâches informationnelles et pédagogiques complexes d'ajustement ou de mise en correspondance des expériences personnelles et des injonctions du collectif. Si notre protocole ne nous permet pas de creuser plus avant ni la dimension biographique ni le lien avec les pratiques de classe, l'entrée par la culture de l'information des enseignants vient interroger leurs capacités d'autonomisation, et non pas seulement leur maîtrise technique, des environnements informationnels. Il s'agit de replacer cette culture de l'information, les pratiques informationnelles individuelles et collectives, dans la culture et la formation professionnelles des enseignants. Plus globalement, nos résultats et les enjeux qu'ils soulèvent sont à prendre en compte dans la formation par ces mêmes enseignants des élèves à l'information.

\section{Bibliographie}

Abric J.C. (1993). «L'étude expérimentale des représentations sociales ». In Jodelet D. (dir.), Les représentations sociales. Paris, Presses universitaires de France.

Adler J. (2010). «La conceptualisation des ressources. Apports pour la formation des professeurs de mathématiques ». In Gueudet G., Trouche L. (dir.), Ressources vives. Le travail documentaire des professeurs en mathématiques. Rennes, Presses universitaires de Rennes et INRP, p. 23-40.

Ball D. L., Cohen D. K. (1996). «Reform by the book: What is - or might be - the role of curriculum materials in teacher learning and instructional reform?». In Educational Researcher, vol. 25, $\mathrm{n}^{\circ}$ 9, p. 6-14.

Barrère A. (2002). Enseignants au travail routines incertaines, Paris, L'Harmattan. 
Baron G.L., Dané E. (2007). «Pédagogie et ressources numériques en ligne : quelques réflexions ». In EPINet [En ligne], disponible sur http://www.epi.asso.fr/revue/articles/a0709c.htm (page consultée le 05/11/2019).

Besnier S., Gueudet G. (2016). «Usages de ressources numériques pour l'enseignement des mathématiques en maternelle : orchestrations et documents ». In Perspectivas da Educação Matemática, vol. 9, n² 21, p. 978-1003.

Bougnoux D. (1995) La communication contre l'information, Paris, Hachette.

Chaudiron S., Ihadjadene M. (2010). «De la recherche de l'information aux pratiques informationnelles ». In Études de communication, ${ }^{\circ} 35$, p. 13-30.

Diekema A.R., Olsen M.W. (2011). «Personal Information Management Practices of Teachers ». In Proceedings of the American Society for Information Science and Technology, vol. $48, \mathrm{n}^{\circ} 1$, p. 1-10.

Diekema A.R., Olsen M.W. (2012). «The notion of relevance in teacher information behavior ». In Proceedings of the American Society for Information Science and Technology, vol. $49, \mathrm{n}^{\circ} 1$, p. $1-9$.

Diekema A.R., Olsen M.W. (2014). «Teacher Personal information management (PIM) practices: Finding, keeping, and Re-Finding information ». In Journal of the Association for Information Science and Technology, vol. 65, $\mathrm{n}^{\circ} 11$, p. 2261-2277.

Dubar C., Tripier P. (2005). Sociologie des professions, Paris, Armand Colin.

Dumouchel G., Karsenti T. (2017). «Mon ami Google : une étude des pratiques des futurs enseignants du Québec en recherche d'information». In La revue canadienne de l'apprentissage et de la technologie, vol. 43, n 2, p. 2-20.

Endrizzi L. (2012). Les technologies numériques dans l'enseignement supérieur, entre défis et opportunités. In Dossier d'actualité veille et analyses [en ligne], $\mathrm{n}^{\circ} 78$, disponible sur http://veille-et-analyses.ens-lyon.fr/DA-Veille/78-octobre-2012.pdf (page consultée le 05/11/2019).

Gardiès C., Fabre I., Couzinet I. (2010). «Re-questionner les pratiques informationnelles ». In Études de communication, $\mathrm{n}^{\circ} 35$, p. 121-132.

Gueudet G., Trouche L. (2008). « Du travail documentaire des enseignants : genèses, collectifs, communautés. Le cas des mathématiques ». In Education et Didactique, vol. 2, n 3 , p. 7-33.

Gueudet G., Trouche L. (2010). « Introduction ». In Gueudet G., Trouche L. (dir.), Ressources vives. Le travail documentaire des professeurs en mathématiques. Rennes, Presses universitaires de Rennes et INRP.

Guyot B. (2009). «Se mouvoir au sein du monde de l'information : comment les personnes parlent de leur activité d'information ». In Études de communication, n 33, p. 101-118. 
Juanals B. (2003). La culture de l'information, du livre au numérique, Paris, Hermès Science Publications.

Loffreda M. (2017). « Gestion des ressources éducatives par les enseignants du secondaire : de l'environnement personnel à l'environnement professionnel ». In Rapport STEF dans le cadre de la convention DNE [en ligne], Laboratoire STEF, ENS Paris-Saclay, disponible sur http://cache.media.eduscol.education.fr/file/ -Sans nom/08/9/LOFFREDA_Rapport_DNE_PIM_gestion_ressources_educatives_060717_816089.pd $\underline{\text { f }}$ (page consultée le 05/11/2019).

Margolinas C., Wozniak F. (2010). «Rôle de la documentation scolaire dans la situation du professeur : le cas de l'enseignement des mathématiques à l'école élémentaire ». In Gueudet G., Trouche L. (dir.), Ressources vives. Le travail documentaire des professeurs en mathématiques. Rennes, Presses universitaires de Rennes et INRP, p. 233-249.

Messaoui A. (2016). «Les pratiques informationnelles individuelles et collectives des enseignants d'un collège rural. Une enquête exploratoire ». In Spiral-E, n ${ }^{\circ}$ 57, p. 55-82.

ministère de l'Éducation nationale (2016). Enquête PROFETIC auprès de 5000 enseignants du second degré : Rapport [en ligne], disponible sur http://cache.media.eduscol.education.fr/file/ETIC_et_PROFETIC/15/4/PROFETIC_2016_Rapport complet 648154.pdf (page consultée le 05/11/2019)

Perrenoud P. (1993). « Sens du travail et travail du sens à l'école ». In Cahiers pédagogiques, $\mathrm{n}^{\circ} 314-315$, p. 23-27.

Remillard J. (2013). « Examining resources and re-sourcing as insights into teaching ». In ZDM, The International Journal of Mathematics Education, vol. 45, $\mathrm{n}^{\circ}$ 7, p. 925-927.

Remillard J., Herbel-Eisenmann B., Lloyd G. (2009). Mathematics teachers at work: Connecting curriculum materials and classroom instruction, New-York, Routledge.

Reverdy C. (2014). Du programme vers la classe : des ressources pour enseigner, Dossier de veille de l'IFÉ [en ligne], $\mathrm{n}^{\circ} 96$, disponible sur: http://veille-et-analyses.ens-lyon.fr/DAVeille/96-novembre-2014.pdf (page consultée le 05/11/2019).

Souchier E., Le Marec J., Jeanneret Y. (2003). Lire, écrire, récrire : objets, signes et pratiques des médias informatisés, Paris, BPI. 